BMJ Open Sport \& Exercise Medicine

\title{
Efficacy of high-volume injections with and without corticosteroid compared with sham for Achilles tendinopathy: a protocol for a randomised controlled trial
}

\author{
Peter Malliaras (D) , ${ }^{1}$ David Connell, ${ }^{1,2}$ Anders Ploug Boesen, ${ }^{3}$ \\ Rebecca S Kearney, ${ }^{4,5}$ Hylton B Menz,, ${ }^{6,7}$ Dylan Morrissey, ${ }^{8,9}$ \\ Shannon E Munteanu, ${ }^{6,7}$ Karin G Silbernagel, ${ }^{10}$ Martin Underwood, ${ }^{4}$ \\ Terry $\mathrm{P}$ Haines ${ }^{1}$
}

To cite: Malliaras $P$, Connell D, Boesen AP, et al. Efficacy of high-volume injections with and without corticosteroid compared with sham for Achilles tendinopathy: a protocol for a randomised controlled trial. BMJ Open Sport \& Exercise Medicine 2021;7:e001136. doi:10.1136/ bmjsem-2021-001136

Accepted 1 October 2021
Check for updates

(C) Author(s) (or their employer(s)) 2021. Re-use permitted under CC BY-NC. No commercial re-use. See rights and permissions. Published by BMJ.

For numbered affiliations see end of article.

Correspondence to Dr Peter Malliaras; Peter.Malliaras@monash.edu

\section{ABSTRACT}

Introduction Achilles tendinopathy (AT) is a common and disabling musculoskeletal condition. First-line management involving Achilles tendon loading exercise with, or without, other modalities may not resolve the problem in up to $44 \%$ of cases. Many people receive injections. Yet there are no injection treatments with demonstrated long-term efficacy. The aim of the trial is to examine the 12-month efficacy of high-volume injection (HVI) with corticosteroid and HVI without corticosteroid versus sham injection among individuals with AT.

Methods and analysis The trial is a three-arm, parallel group, double-blind, superiority randomised controlled trial that will assess the efficacy of HVI with and without corticosteroid versus sham up to 12 months. We will blockrandomise 192 participants to one of the three groups with a 1:1:1 ratio, and both participants and outcome assessors will be blinded to treatment allocation. All participants will receive an identical evidence-based education and exercise intervention. The primary outcome measure will be the Victorian Institute of Sport Assessment - Achilles (VISA-A) at 12 months post-randomisation, a validated, reliable and disease-specific measure of pain and function. Choice of secondary outcomes was informed by core outcome domains for tendinopathy. Data will be analysed using the intention-to-treat principle.

Ethics and dissemination Ethics approval was obtained via the Monash University Human Ethics Committee (no: 13138). The study is expected to be completed in 2024 and disseminated via peer review publication and conference presentations.

Trial registration number Australia and New Zealand Clinical trials registry (ACTRN12619001455156)

\section{INTRODUCTION}

Achilles tendinopathy (AT) is a common, painful and disabling musculoskeletal condition characterised by local tissue pathology, swelling and activity-related pain. A 2011

\section{Key messages}

What is already known

- There are no injection treatments for Achilles tendinopathy with demonstrated long-term efficacy compared with placebo or sham.

- The high-volume injection (HVI) includes local anaesthetic and saline with or without corticosteroid.

- There is efficacy compared with sham at 6 months for the injection with but not without corticosteroid.

What are the new findings

- The proposed trial will address a knowledge gap by evaluating the efficacy of the HVI with and without corticosteroid versus sham for Achilles tendinopathy over 12 months.

- This research has the potential to provide evidence to support or refute the HVI in clinical practice for people with non-responsive Achilles tendinopathy

Dutch general practice study among 57725 adults (21-60 years) reported an Achilles tendinopathy incidence of 2.35 per 1000 GP registered patients, ${ }^{1}$ with $35 \%$ of cases not involving sport. ${ }^{1} \mathrm{Up}$ to $18 \%$ of running injuries are related to Achilles tendinopathy. ${ }^{2}$ The cumulative prevalence in the general population has been reported to be $6 \% .^{3}$ Achilles tendinopathy can severely impact quality of life (particularly mobility, usual activity and pain domains), is associated with reduced work capacity in $38 \%$ of people affected and accounts for substantial healthcare costs. ${ }^{4}$

The aetiology of Achilles tendinopathy is multifactorial $^{5}$ and the imbalance between load demands placed on the tendon and its ability to remodel is considered a major factor. ${ }^{6}$ Other factors that are thought to increase tendon load or influence the 
remodelling capacity of the tendon may increase the risk of AT, including reduced plantarflexor strength, genetic profile and metabolic factors (eg, elevated cholesterol or diabetes). ${ }^{57}$ Once established, Achilles tendon tissue changes include matrix degradation (characterised by inferior quality and disorganised collagen), accumulation of hydrophilic proteoglycan molecules that increase bound water causing swelling, as well as ingrowth of blood vessels and nerves. ${ }^{6}$ The ingrowth of blood vessels and nerves from the deeper lying Kager's fat pad is considered a potential pain mechanism in $\mathrm{AT}^{8}$ because biochemicals involved in pain transmission (eg, substance $\mathrm{P}$ ) are released by in-growing nerve tissue. ${ }^{910}$ Thus, the ingrowth of nerves from Kager's fat pad is a potential treatment target.

Achilles tendon loading exercise is recommended as a first-line treatment for Achilles tendinopathy in clinical practice guidelines and expert narratives. ${ }^{11}{ }^{12}$ However, up to $44 \%$ of people may not respond to exercise treatments for AT. ${ }^{13}$ Up to $60 \%$ of people with AT have continued pain and disability after 5 years despite exercise interventions, and $48 \%$ will seek additional treatment including injections and surgery. ${ }^{14}$

There are currently no injection treatments with demonstrated long-term efficacy. Corticosteroid injections demonstrate short-term efficacy in improving tendinopathy pain and function, but there is welldocumented symptom recurrence in the longer term. ${ }^{15-17}$ Other injections in clinical use for AT, including polidocanol, aprotinin and platelet-rich plasma injections, have been shown to be no more effective than placebo or sham, ${ }^{18}$ although a network meta-analysis found benefits compared with wait-and-see. ${ }^{19}$ An injection option that demonstrated efficacy compared with placebo or sham is likely to reduce the substantial and long-standing pain and disability, as well as costs associated with managing AT.

The high-volume injection (HVI) is a relatively new injection used to manage $\mathrm{AT}^{20}$ This was first introduced in 2008. ${ }^{20}$ HVI typically consists of a corticosteroid and local anaesthetic in a $50 \mathrm{~mL}$ injection. The fluid is injected between Kager's fat and the Achilles tendon and is hypothesised to disrupt neurovascular ingrowth from Kager's fat to the Achilles tendon that is believed to be a key source of pain in $\mathrm{AT}^{21}$ The proposed mechanisms of HVI include trauma or ischaemic pressure from the large volume of fluid which may impair nerve function and pain transmission ${ }^{22}$ and anti-inflammatory effect from the corticosteroid.

There have been several case series reporting positive effects of the HVI for AT $^{2023}$ but limited randomised trials until recently. Preliminary data from our team ${ }^{25}$ (2017, n=19 in each group) demonstrated benefit for primary pain and function outcome compared with sham injection at 6,12 and 26 weeks. ${ }^{25}$ In a follow-up trial ${ }^{26}$ (2019, n=14 in each group), we compared HVI with and without corticosteroid and found that the short-term benefit ( 6 and 12 weeks) was absent but the medium-term benefit (26 weeks) remained when the corticosteroid was removed. A 2020 randomised trial ${ }^{27}(\mathrm{n}=38 \mathrm{HVI}$ without corticosteroid and $n=41$ sham) compared the HVI without corticosteroid and sham and found no differences in primary pain and function outcome at any timepoint up to 26 weeks (2, 6, 12 and 26 weeks). It is important to determine whether the effects of HVI with corticosteroid are maintained over 12 months or whether there is symptom recurrence as observed with other corticosteroid injections for tendinopathy. ${ }^{15}$ Further, the medium-term and long-term efficacy of HVI without corticosteroid is uncertain.

The primary aim of this trial is to examine the efficacy of HVI with corticosteroid and HVI without corticosteroid versus sham injection among individuals with AT at 12 months.

\section{METHODS AND ANALYSIS Study design}

This is is a three-arm, parallel group, double-blind, superiority randomised controlled trial with a 12-month follow-up. Participants will be block-randomised into sham injection, HVI with corticosteroid or HVI without corticosteroid groups with a 1:1:1 ratio. Participants and outcome assessors will be blinded to treatment allocation. All participants will receive an identical evidence-based education and exercise intervention, which removes ethical concerns of withholding treatment. This protocol is reported in accordance with the 2013 SPIRIT guidelines. $^{28}$

\section{Setting}

The trial is based in a large radiology clinic in central Melbourne, Australia (Imaging at Olympic Park (IOP)). This setting in a central location allows implementation of a previously successful strategy to recruit people with AT from throughout metropolitan Melbourne, and there is a large network of physiotherapists, sports physicians and surgeons who usually refer people with AT to IOP.

\section{Sample size}

The sample size was determined based on our primary endpoint, the Victorian Institute of Sport Assessment Achilles (VISA-A) scale (0-100 points). Minimal clinically important difference (MCID) has not been determined for midportion AT, but prior trials have estimated MCID to be 10 points. ${ }^{2529}$ Based on our preliminary data, ${ }^{25}{ }^{26}$ we assume a 10-point benefit of HVI compared with sham at 12 months. Assuming a SD from our preliminary trial of $15.6,{ }^{25} 51$ participants per group will provide $80 \%$ power to detect a 10-point difference between any of the three trial arms (type I error rate split to $1.67 \%$ for each of the three comparisons). We will recruit 64 participants per trial arm to allow for attrition of up to $20 \%$ at 12 months.

\section{Eligibility criteria}

Inclusion and exclusion criteria are shown in table 1. 
Table 1 Inclusion and exclusion criteria

\begin{tabular}{|c|c|}
\hline Inclusion criteria & Exclusion criteria \\
\hline $\begin{array}{l}\text { 1. Previously failed any treatment for their Achilles } \\
\text { tendinopathy (eg, exercise, medication, advice provided } \\
\text { by a health professional) } \\
\text { 2. } \geq 18 \text { years of age } \\
\text { 3. Mid-portion Achilles pain for } \geq 3 \text { months } \\
\text { 4. Primary complaint of mid-portion Achilles pain on one or } \\
\text { both lower limbs } \\
\text { 5. VISA-A <75 points } \\
\text { 6. Clinical diagnosis of mid-portion Achilles tendinopathy } \\
\text { based on gradual onset pain in the mid-portion Achilles } \\
\text { and midportion Achilles pain during or after Achilles } \\
\text { tendon loading activities (eg, walking, running) } \\
\text { 7. Normal ankle joint examination, especially passive } \\
\text { plantar-flexion } \\
\text { 8. Ultrasound imaging pathology including one or more of } \\
\text { the following features: hypoechoic regions and/or Doppler } \\
\text { signal indicative of vascularisation }\end{array}$ & $\begin{array}{l}\text { 1. Prior Achilles tendon surgery or rupture on the most } \\
\text { symptomatic Achilles tendon } \\
\text { 2. Other ankle conditions including impingement syndrome, } \\
\text { insertional Achilles tendinosis, Achilles paratenonitis without } \\
\text { tendinopathy } \\
\text { 3. Inflammatory arthropathy (eg, ankylosing spondylitis) } \\
\text { 4. Neurological disorders (ie, Parkinson's syndrome) } \\
\text { 5. Inherited connective tissue disorders (ie, Ehlers-Danlos } \\
\text { syndrome, Marfan's syndrome) } \\
\text { 6. Use of fluoroquinolone antibiotics within the previous } \\
\text { 2years } \\
\text { 7. Injection of local anaesthetic, corticosteroid, platelet-rich } \\
\text { plasma or other pharmaceutical agent into the Achilles } \\
\text { tendon or surrounding area within the previous } 3 \text { months } \\
\text { 8. Any medical reason that, in the opinion of the investigators, } \\
\text { makes the participant unsuitable for inclusion } \\
\text { 9. Serious mental health problem (ie, major depressive or } \\
\text { psychotic disorders requiring medical attention/untreated) } \\
\text { that would preclude adherence to study or treatment } \\
\text { protocols } \\
\text { 10. Known allergies or hypersensitivity to the study drugs } \\
\text { 11. Needle phobia or a blood clotting disorder } \\
\text { 12. Pregnancy }\end{array}$ \\
\hline
\end{tabular}

\section{Recruitment and retention}

Recruitment channels include an advertising campaign in local newspapers, flyers advertising the trial placed at local community and sporting clubs, retirement villages and senior citizen centres, via a large network of clinical partners who refer people with AT to IOP, and via a paid social media campaign, including Facebook and Twitter. To improve retention, participants receive a $\$$ A100 shopping voucher on completion of 26-week and 52-week outcomes to reimburse them for costs incurred (time and travel).

\section{Procedure}

Respondents to newspaper, flyer and social media advertising, and potential participants alerted to the trial via clinical partners, will initially be screened via telephone (when they call the researchers, or receive a call back to social media, email or text contact). Potentially eligible participants will be provided with electronic study information and given at least 24 hours to determine whether they would like to proceed. Those who contact the researchers interested in participating will be booked into a baseline session at IOP. A researcher and ultrasonographer blinded to treatment allocation will assess eligibility (figure 1). Eligible participants will be asked to provide written consent and complete the baseline assessment prior to randomisation.

\section{Baseline assessment}

Standard demographic characteristics (age, sex, weight, height, body mass index, ethnicity), healthcare history (medical history, surgical history, current medications) and specific AT history (site, duration, previous treatment) will be recorded at baseline. The following measures will also be assessed at baseline: (1) Achilles tendon pain map: via a computer application; (2) Pain Detect: a validated questionnaire used to detect people who have neuropathic pain ${ }^{30}$; (3) pressure pain thresholds: assessing local (Achilles tendon) and diffuse (lateral elbow) hyperalgesia; (4) expectancy/credibility: participants will be asked about how logical and successful they feel the treatment they have received will be, as well as their confidence in the treatment. ${ }^{31}$

\section{Randomisation}

Eligible participants will be randomly assigned to one of the three treatment arms with a 1:1:1 ratio using computer-generated permuted blocks of variable size $(4$, 8 and 12). This process will be undertaken remotely using third-party web-based randomisation (Griffith University, Gold Coast, Australia) to ensure allocation concealment. For eligible participants, a radiology nurse will use the web-based service to determine treatment allocation. Only the nurse who prepares the injection (figure 1) will be aware of treatment allocation and they will be instructed to minimise interaction with the participants.

\section{Blinding}

Procedures to ensure participant blinding include:

1. Preparing both the HVI and sham injections for all participants. The HVI consists of five $10 \mathrm{~mL}$ syringes, the first with exactly $10 \mathrm{~mL}$ of $0.5 \%$ bupivacaine hydrochloride and $25 \mathrm{mg}$ of hydrocortisone, followed by four syringes with $10 \mathrm{~mL}$ each of normal saline. All five syringes will be prepared regardless of group allocation, ensuring preparation time is identical. 


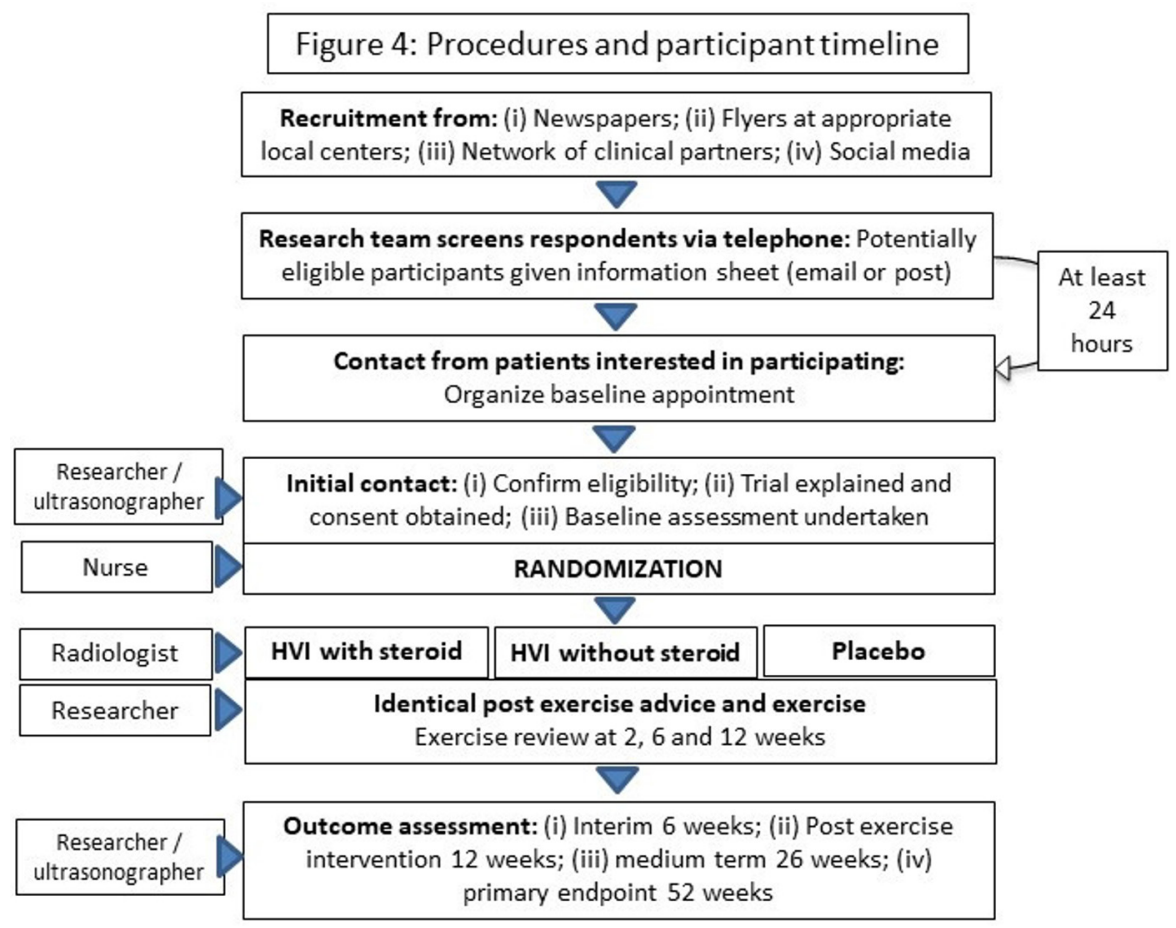

Figure 1 Participant flow through the trial.

2. Using identical injection procedures for all groups. All five syringes will also be injected so treatment time is identical. For the sham group, the excess injectant will be diverted away from the patient and to a $50 \mathrm{~mL}$ syringe via a three-way stop cock (figure 2)

3. A sleeve placed over the first syringe, so the radiologist is not able to detect whether the syringe contains corticosteroid (the corticosteroid changes the colour of the injectant). Piloting has indicated that the colour of the injectant is not clearly visible from the thin injecting tube. The radiologist will be aware when sham is the allocated group.

Unblinding will only be permissible if one of the participants experiences a serious adverse event that may be

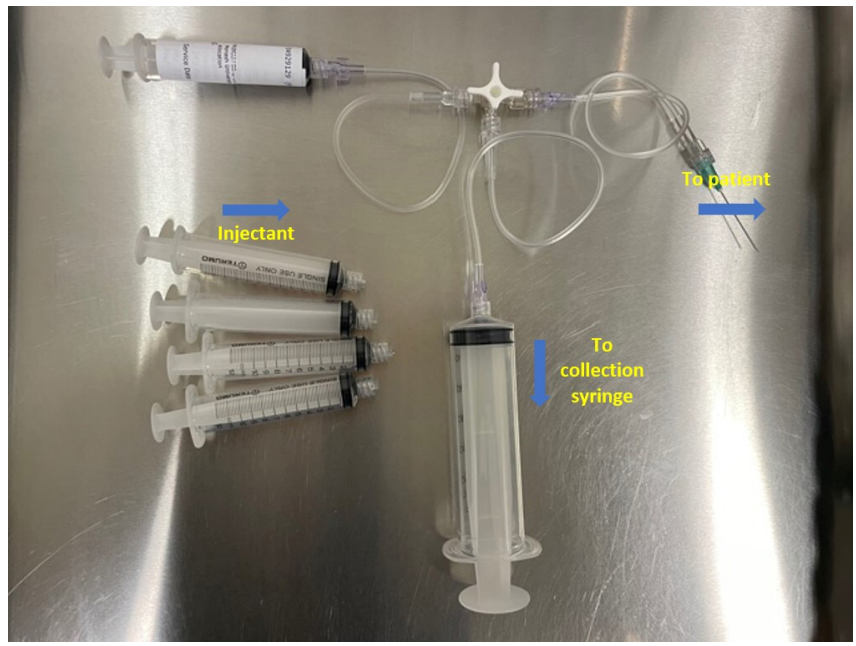

Figure 2 Three-way stop cock is used to divert the injectant into the $50 \mathrm{~mL}$ collection syringe for the sham group. related to one of the medications administered. If this occurs, a member of the trial management team (who is not involved in data collection) will unblind the participant and notify the treating medical staff.

\section{Three injection groups}

In participants with bilateral symptoms, the most symptomatic side (volunteered by the patient on questioning, or the right side if they cannot define the more symptomatic side) will be injected. All participants will receive a single injection (see figure 3) that will be delivered by an experienced musculoskeletal radiologist while participants are lying prone. Immediately post-injection, participants will be asked which injection they believe they received (they are able to answer whether they think it is intervention, sham or that they do not $\mathrm{know}^{32}$ ) to assess the success of participant blinding.

1. High-volume injection with corticosteroid: The HVI will be injected into the interface between Kager's fat and the Achilles tendon. The local anaesthetic and the corticosteroid is injected followed by the four saline syringes by using a connecting tube (allows consecutive syringes to be attached). The position of the needle will be monitored continuously by ultrasound and the needle will be moved gently across the anterior aspect of the tendon to ensure uniform effect over the pathological area.

2. Tendon sheath injection without corticosteroid: This injection is identical to the HVI with corticosteroid, but the first syringe will only contain exactly $10 \mathrm{~mL}$ of $0.5 \% \mathrm{bu}-$ pivacaine hydrochloride (no corticosteroid).

3. Sham injection: The sham injection consists $2-3 \mathrm{~mL}$ of $0.5 \%$ bupivacaine hydrochloride and will be injected 


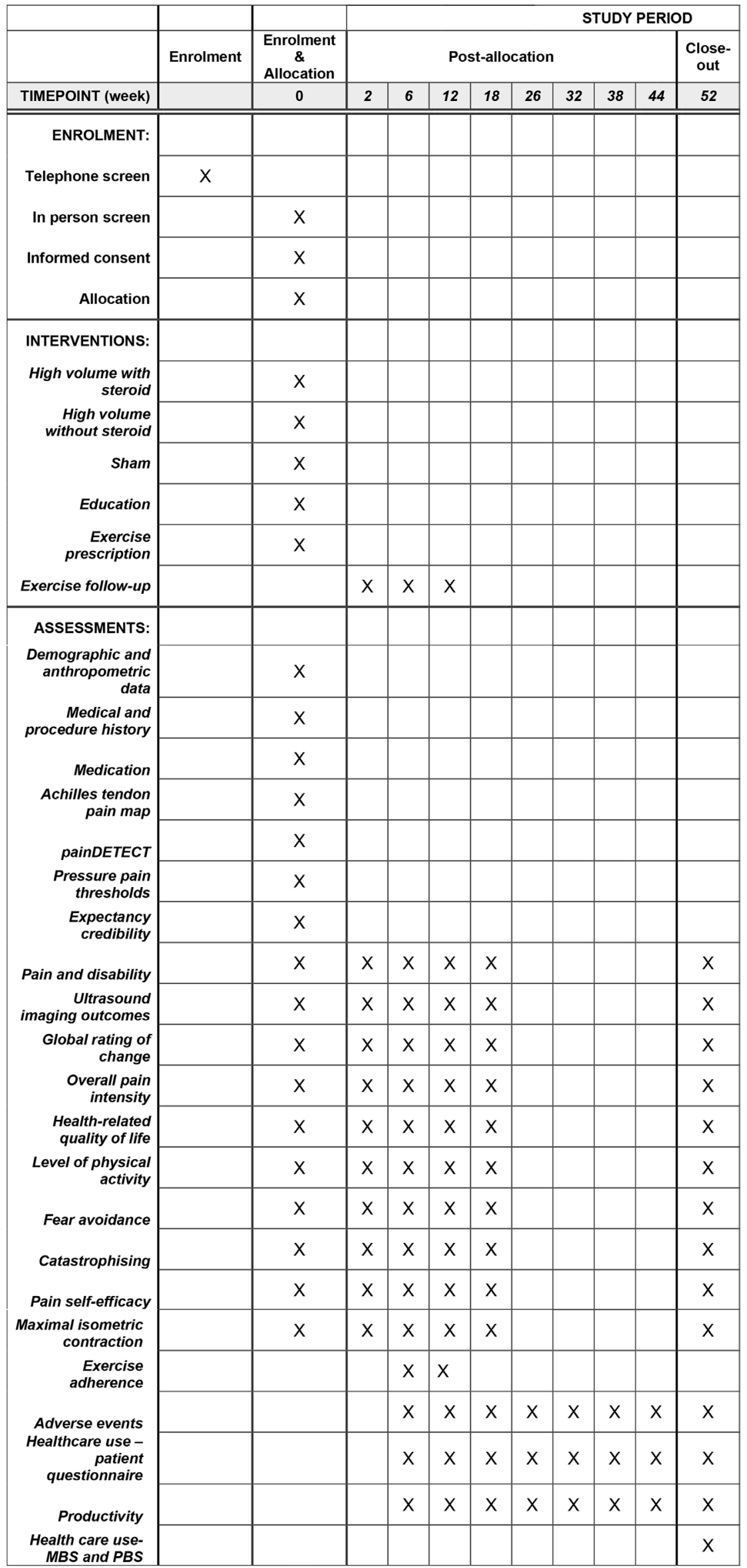

Figure 3 Schedule of enrolment, interventions and assessments. 
under image guidance by the same expert radiologist, deep to the tendon and away from the interface between Kager's fat and the Achilles tendon (at last $10 \mathrm{~mm}$ away). The sham injection will result in a numbing effect, like the HVI.

\section{Post-injection treatment}

Immediately after the injection, an appropriately trained researcher (figure 1) who is blind to treatment allocation will deliver identical education and a 12-week exercise programme to all participants (see table 2 Figure 3), based on prior HVI work. ${ }^{20}{ }^{25}$ This includes an evidencebased and progressively loaded exercise protocol for AT, ${ }^{3334}$ education about physical activity modification and resumption, and advice about progressing and regressing exercise load, based on a pain monitoring model. ${ }^{35}$ The researcher will also provide education about AT (pathology, pain, risk factors, prognosis, treatments, recovery times) and post-injection advice. The information provided to patients (in printed form) at baseline is shown in online supplemental file 1 and the exercise videos provided are shown in online supplemental file 2. Participants will be reviewed by the researcher on four follow-up occasions-1week (brief phone call to check post-injection response and whether exercise was commenced), 2 weeks via teleconference and 6 weeks in person (modify the exercise as required, eg, progress load, modify technique), and at 12 weeks-to provide evidence-based advice about continuing exercise beyond the intervention period. ${ }^{25}$

To ensure participants are supported and to optimise uptake of the 12-week exercise programme, they will be able to email or call the researchers with questions and will be provided with custom-produced basic online exercise videos and education. Participants will be advised to refrain from using other physical therapy interventions and non-steroidal anti-inflammatory medications but will be allowed to take paracetamol (up to $4 \mathrm{~g}$ /day) for pain relief as required.

\section{Intervention fidelity}

The fidelity of each intervention (injections, education and exercise) will be monitored at regular intervals (at least every 8 to 10 injections (minimum of 19 assessments throughout the trial)) by a member of the trial management group who is not involved in data collection or analysis. Radiologists and physiotherapists delivering the treatment will be provided with feedback and strategies to improve fidelity as appropriate.

\section{Outcome measures}

Outcomes are either self-report or performed by appropriately trained researchers or ultrasonographer blinded to the treatment allocation. Outcome assessment will occur at baseline, 6, 12, 26 and 52 weeks (see figure 3). All follow-ups will occur at the same imaging facility as baseline assessment. All data collected will be pseudoanonymised (retaining code file) and stored on a secure cloud-based server. Participants will complete self-report outcomes in relation to their most symptomatic Achilles tendon if they have bilateral AT.

1. Primary outcome: Pain and function will be assessed with the VISA-A, a well-validated, reliable and diseasespecific tool. ${ }^{36}$ This outcome includes pain, function and activity domains that are clinically relevant to patients with AT. Scores range from 0 to 100 , with 100 indicating no symptoms or function/activity limitations. The final two question of the VISA-A were designed for sporting populations and will be modified in our study to relate to our mixed sporting and nonsporting population. Question 7 will be modified from "Are you currently undertaking sport or other physical activity?" to "Are you currently undertaking sport or other physical activity, including walking?”. Question 8 asks about pain and disability during Achilles tendon loading sport. 'Achilles tendon loading sport' will be changed to 'weight-bearing activity'. Both versions of question 8 will be included so that responsiveness of both versions of the VISA-A can be evaluated.

2. Secondary outcomes:

a. Achilles tendon thickness and Doppler signal: assessed with ultrasound imaging using reliable methods. ${ }^{37}$ Doppler signal will be assessed by quantifying pixel count. ${ }^{38}$ This outcome is important to assess whether tendon adaptation and change in Doppler signal is a potential mechanism of the HVI.

b. The Global Rating of Change Scale: an 11-point scale in which the participant is asked to rate their perceived overall change in their AT condition from the time that they began the study until the present, as Worse, No Change or Better. If they indicate worse, the patient will then be asked how much worse on a 5-point scale from Very Much Worse to Slightly Worse, and if they are better, they will be asked how much better on a 5-point scale from Slightly Better to Very Much Better. ${ }^{39}$

c. Overall pain intensity: measured using the $100 \mathrm{~mm}$ visual analogue scale (VAS), participants will rate the worst pain during the last week (zero=no pain; $100=$ worst pain possible).

d. Health-related quality of life: measured with the EQ-5D-5L, a validated and reliable tool, ${ }^{40}$ including five domains (mobility, self-care, usual activities, pain/discomfort and anxiety/depression), and a rating of overall health state from 0 (worst health state imaginable) to 100 (best imaginable health state) using a VAS.

e. The level of physical activity in the previous week: evaluated using the 7-day Recall Physical Activity Questionnaire, a valid and reliable tool. ${ }^{41}$ Participants will be asked to recall time spent sleeping and doing physical activity (work, leisure, household activities) over the past 7 days.

f. Fear avoidance and catastrophising: the Tampa Kinesiophobia Scale ${ }^{42}$ and Pain Catastrophising Scales ${ }^{43}$ are validated questionnaires used to mea- 
sure fear avoidance and pain catastrophising, respectively.

g. Pain self-efficacy: assessed with the pain self-efficacy questionnaire (PSEQ). ${ }^{44}$ The PSEQ measures how confident a patient is in undertaking a range of activities despite their pain.

h. Maximal voluntary isometric contraction (MVIC) and rate of force development (RFD) ${ }^{45}$ : seated calf raise RFD and MVIC. Standard strength (MVIC and RFD) tests will be performed on a custom chair equipped with a force plate that measures plantarflexor force. Participants will have an adequate warm-up (10 repetitions of calf raises with the knee bent and knee straight) and then perform 4 practice and 2 recorded trials. The instructions will be "push as hard and fast as possible".

3. Other secondary outcomes: selected outcomes will be assessed at every 6 weeks from weeks 6 to 52 via online questionnaire (if not coinciding with an assessment visit time).

Adverse event: an adverse event is defined as any unfavourable or unintended diagnosis, sign, symptom, or disease associated with the study which may or may not be related to the intervention (eg, tendon rupture, fall, injury or change in medical status) and prevents participation in the intervention for at least seven consecutive days. Participants will record what each adverse event was (eg, tendon rupture), impact on their ability to undertake the intervention and for how long.

Serious adverse events: defined as an event related or not related to the intervention that results in death, life-threatening complication, hospitalisation, surgery, permanent or temporary physical disability, congenital abnormalities, or any findings the CI and/or research team feel can lead to significant health hazards. Examples of serious adverse events include but are not limited to surgery for tendon rupture and heart attack requiring overnight hospitalisation.

\section{Reporting of adverse events}

If the adverse event is not defined as serious, the adverse event is recorded in the trial file and the participant is followed up by the research team. All serious adverse events will be recorded in the participant's trial file and reported to the chief investigator immediately. The chief investigator will let the trial sponsor know within 24 hours, as well as data monitoring, trial management and steering committees (described later).

\section{Action plan for addressing adverse events}

If the adverse event occurs at the trial setting (IOP, Melbourne), then the centre policy for adverse events will be followed. If the adverse event occurs outside the trial setting, then the participants will be advised to see their GP or go to the emergency department for appropriate management. All the treatment costs will be covered by the sponsor's insurance. b. Exercise adherence: participants will record the number of exercise sessions completed each week over the previous 4 weeks (adherence is the percentage of prescribed sessions that are completed). Adherence will be categorised as (i) poor, $<25 \%$; (ii) moderate, $25 \%$ to $50 \%$; (iii) good, $>50 \%$ to $75 \%$; or (iv) excellent, $>75 \% .{ }^{46}$ Exercise adherence will be measured at 6 and 12 weeks.

c. Healthcare use: Medicare Benefits Schedule (www. mbsonline.gov.au/) and Pharmaceutical Benefits Schedule (www.pbs.gov.au) data will be extracted to measure use of subsidised healthcare services. The use of other health service and co-interventions will be measured by asking for a yes/no response followed by a phone call.

Productivity: productivity (including absenteeism and presentism) will be measured using the 'iMTA Productivity Cost Questionnaire'. ${ }^{47}$

\section{Statistical methods}

Data will be double entered to minimise errors and coded to ensure blinding of the statistician undertaking the analyses. In participants with bilateral symptoms, the most symptomatic side (or the right side if they cannot define the more symptomatic side) will be analysed to maintain independence of data. Statistical tests will be two-tailed with statistical significance level set at 0.0167 for each of the three pairs of comparisons of trial arms. We will also analyse the primary outcome with alpha set at 0.05 and provide this as a supplemental file for future systematic reviewers. All randomised patients will be included in the analysis (ie, intention-to-treat) for primary and secondary outcomes (except for safety outcomes). We will also undertake secondary per-protocol analyses. Demographic characteristics (eg, age, gender) and other baseline measurements (eg, duration of symptoms) will be reported by treatment arm. Between-group differences in the primary and secondary outcomes measures will be compared at 12, 26 and 52 weeks, with the primary outcome being at 52 weeks. Continuously scored outcome measures will be analysed using linear mixed models with adjustments for baseline scores and variables that are found to influence the outcome. Ordinal scaled data will be analysed using non-parametric tests and modelled with proportional odds regression adjusted for repeated assessment of subjects. Dichotomous outcome measures will be compared using relative risk, risk difference and number needed to treat using generalised estimating equations. Sensitivity to missing data will be assessed using multiple imputation models incorporating predictive baseline and post-baseline variables.

\section{Economic analysis}

Alongside the main trial, there will be a parallel, trialbased cost-utility analysis. This economic analysis will consider the incremental cost of each of the intervention conditions compared with the sham for gaining one quality-adjusted life year. Quality-adjusted life years will 
be calculated using an area under the curve approach on EQ-5D-5L utility scores collected at each assessment. These scores will be generated using the Dolan crosswalk approach. ${ }^{48}$ A societal perspective will be taken by calculating healthcare and productivity costs incurred by participants. Valuation of health services costs will be based on standard rates published by the Australian government (National Weighted Activity Unit costs for hospitalisations (The Independent Hospital Pricing Authority website, https://www.ihpa.gov.au/what-we-do/ national-weighted-activity-unit-nwau-calculators-201516), Medical Benefits Schedule and Pharmaceutical Benefits Schedule prices for other subsidised health costs, market prices for other costs). Bootstrap resampling will be used to generate CIs for cost-utility estimates and for construction of acceptability curves. One-way sensitivity analyses will also be conducted to examine the impact of variation in key analysis inputs.

\section{Trial governance}

A trial management group (TMG) will be convened and meet up to quarterly. In addition to this, a data safety management board (DSMB) and a trial steering committee (TSC) will also be convened. The TMG is composed of the research team and will meet quarterly to oversee trial management. The DSMB will include an independent multidisciplinary group consisting of two biostatisticians and clinical experts (injection and exercise), who will monitor the safety and compliance issues related to the trial. The TSC will include a core member of the TMG, expert clinicians (injections and exercise), and at least one lay representative and will be responsible for issues such as recruitment, trial management and safety concerns. The TSC and DSMB will meet once to twice a year. The first review of safety (undertaken by the full TMG) will occur when 20 participants have completed the 12-week assessment and then every 6 months until the conclusion of the trial.

\section{Patient and public involvement}

Patients will be involved in the trial TSC from inception and will have a role in developing the research design, outcome measures and recruitment strategy.

\section{ETHICAL CONSIDERATIONS}

This study protocol was granted ethics approval by the Monash University Human Ethics Committee (no: 13138). Any amendments to the protocol will be reviewed by this ethics committee and communicated to the trial funder and trial registry.

\section{DISSEMINATION}

The findings of this trial will be presented at national and international conferences and submitted to a peer review journal for publication. Dissemination assets will be created, including audience-specific media releases and infographics, and disseminated to stakeholders including patients and clinicians.

\section{DISCUSSION}

AT is common and disabling. As many as up to $44 \%$ of people with AT fail to recover with conservative first-line treatment ${ }^{133749}$ and are offered other treatments including injections. ${ }^{14}$ Despite the common use of injections, there are currently no injection treatments for AT with demonstrated efficacy. Although corticosteroid injections have demonstrated short-term efficacy, there may be recurrence of symptoms for some people in the longer term. ${ }^{15}$ The lack of an efficacious injection for AT may prolong disability for many people who are affected and significantly increase healthcare use and costs. HVI may be an effective treatment for AT but has not been adequately tested. The proposed trial will address this knowledge gap by evaluating the efficacy of the HVI with and without corticosteroid versus sham for AT over 12 months. This research has the potential to enhance recovery of patients thereby improving clinical outcomes and reduce societal costs by reducing failed injection treatments and ongoing disability.

\section{LIMITATIONS}

The radiologist is not blind to the sham interventions, but we will implement measures including a standardised script to ensure that all participants have comparable interventions with this caregiver. It is possible that people who have had a corticosteroid injection close to 3 months ago and up to a year prior may still have long-term adverse events from this injection that influences our findings. These effects are likely to be equal across groups given the randomisation process. A potential limitation to external validity is the single-centre design; however, this is appropriate for the efficacy paradigm adopted in this trial.

\section{Author affiliations \\ ${ }^{1}$ School of Primary and Allied Health Care, Monash University, Frankston, Victoria, Australia \\ 2Imaging @ Olympic Park, Melbourne, Victoria, Australia \\ ${ }^{3}$ Department of Orthopaedic Surgery, Hvidovre Hospital, Copenhagen, Denmark \\ ${ }^{4}$ Warwick Medical School, University of Warwick, Coventry, UK \\ ${ }^{5}$ University Hospitals of Coventry and Warwickshire, Coventry, UK \\ ${ }^{6}$ Discipline of Podiatry, School of Allied Health, Human Services and Sport, La Trobe \\ University, Melbourne, Victoria, Australia \\ ${ }^{7}$ La Trobe Sport and Exercise Medicine Research Centre, La Trobe University, Melbourne, Victoria, Australia \\ ${ }^{8}$ Sports and Exercise Medicine, Queen Mary University of London, London, UK \\ ${ }^{9}$ Physiotherapy Department, Barts Health NHS Trust, London, UK \\ ${ }^{10}$ Department of Physical Therapy, University of Delaware, Newark, DE, USA}

Contributors All authors were involved in developing the methods for this trial and writing the protocol manuscript. PM is the guarantor.

Funding This research has been funded by a National Health and Medical Research Council (NHMRC) Project Grant (APP1164268).

Disclaimer The funder does not/will not have any role in study design, data collection, analysis or interpretation for this trial.

Competing interests $\mathrm{DC}$ is an interventional radiologist who receives pay for interventional procedures such as Achilles tendon injections as part of his work. MU is chief investigator or co-investigator on multiple previous and current research grants from the UK National Institute for Health Research, Arthritis Research UK and is a co-investigator on grants funded by the Australian NHMRC. He is an NIHR Senior Investigator emeritus. He has received travel expenses for speaking at conferences from the professional organisations hosting the conferences. He is a director and shareholder of Clinvivo Ltd that provides electronic data collection for 
health services research. He is part of an academic partnership with Serco Ltd, funded by the European Social Fund, related to return to work initiatives. He is a co-investigator on three NIHR-funded studies receiving additional support from Stryker Ltd. He has accepted honoraria for teaching/lecturing from consortium for advanced research training in Africa. Until March 2020, he was an editor of the NIHR journal series, and a member of the NIHR Journal Editors Group, for which he received a fee. RSK is chief investigator or co-investigator on multiple previous and current research grants from the UK National Institute for Health Research (NIHR) and Arthritis Research UK member. RSK is a member of the UK National Institute for Health Research (NIHR) Health Technology Assessment Clinical Evaluation and Trials board, NIHR Integrated Clinical Academic Doctoral panel, chair of the NIHR Research for Public Benefit board and holder of a NIHR Fellowship award.

Patient and public involvement Patients and/or the public were involved in the design, or conduct, or reporting, or dissemination plans of this research. Refer to the Methods section for further details.

Patient consent for publication Not applicable.

Provenance and peer review Not commissioned; externally peer reviewed.

Data availability statement Data will be made available on request at the completion of the trial.

Open access This is an open access article distributed in accordance with the Creative Commons Attribution Non Commercial (CC BY-NC 4.0) license, which permits others to distribute, remix, adapt, build upon this work non-commercially, and license their derivative works on different terms, provided the original work is properly cited, appropriate credit is given, any changes made indicated, and the use is non-commercial. See: http://creativecommons.org/licenses/by-nc/4.0/.

ORCID iD

Peter Malliaras http://orcid.org/0000-0001-5879-4139

\section{REFERENCES}

1 de Jonge S, van den Berg C, de Vos RJ, et al. Incidence of midportion Achilles tendinopathy in the general population. $\mathrm{Br} \mathrm{J}$ Sports Med 2011;45:1026-8.

2 Rolf C, Movin T, Etiology MT. Etiology, histopathology, and outcome of surgery in achillodynia. Foot Ankle Int 1997;18:565-9.

3 Kujala UM, Sarna S, Kaprio J. Cumulative incidence of Achilles tendon rupture and tendinopathy in male former elite athletes. Clin J Sport Med 2005;15:133-5.

4 Sleeswijk Visser TSO, van der Vlist AC, van Oosterom RF, et al. Impact of chronic Achilles tendinopathy on health-related quality of life, work performance, healthcare utilisation and costs. BMJ Open Sport Exerc Med 2021;7:e001023.

5 Malliaras P, O'Neill S. Potential risk factors leading to tendinopathy. Apunts Medicina de l'Esport 2017;52:71-7.

6 Magnusson SP, Langberg $\mathrm{H}$, Kjaer M. The pathogenesis of tendinopathy: balancing the response to loading. Nat Rev Rheumatol 2010;6:262-8

7 van der Vlist AC, Breda SJ, Oei EHG, et al. Clinical risk factors for Achilles tendinopathy: a systematic review. Br J Sports Med 2019;53:1352-61.

8 Alfredson $\mathrm{H}$, Öhberg L, Forsgren S. Is vasculo-neural ingrowth the cause of pain in chronic Achilles tendinosis? Knee surgery, sports traumatology, arthroscopy 2003;11:334-8.

9 Andersson G, Backman LJ, Scott A, et al. Substance P accelerates hypercellularity and angiogenesis in tendon tissue and enhances paratendinitis in response to Achilles tendon overuse in a tendinopathy model. Br J Sports Med 2011;45:1017-22.

10 Bjur D, Alfredson H, Forsgren S. The innervation pattern of the human Achilles tendon: studies of the normal and tendinosis tendon with markers for general and sensory innervation. Cell Tissue Res 2005;320:201-6.

11 Martin RL, Chimenti R, Cuddeford T, et al. Achilles pain, stiffness, and muscle power deficits: midportion Achilles tendinopathy revision 2018. J Orthop Sports Phys Ther 2018;48:A1-38.

12 Scott A, Docking S, Vicenzino B, et al. Sports and exercise-related tendinopathies: a review of selected topical issues by participants of the second International Scientific Tendinopathy Symposium (ISTS) Vancouver 2012. Br J Sports Med 2013;47:536-44.

13 Sayana MK, Maffulli N. Eccentric calf muscle training in non-athletic patients with Achilles tendinopathy. J Sci Med Sport 2007;10:52-8.

14 van der Plas A, de Jonge S, de Vos RJ, et al. A 5-year follow-up study of Alfredson's heel-drop exercise programme in chronic midportion Achilles tendinopathy. Br J Sports Med 2012;46:214-8.

15 Coombes BK, Bisset L, Vicenzino B. Efficacy and safety of corticosteroid injections and other injections for management of tendinopathy: a systematic review of randomised controlled trials. Lancet 2010;376:1751-67.

16 Fredberg U, Bolvig L, Pfeiffer-Jensen $\mathrm{M}$, et al. Ultrasonography as a tool for diagnosis, guidance of local steroid injection and, together with pressure algometry, monitoring of the treatment of athletes with chronic jumper's knee and Achilles tendinitis: a randomized, double-blind, placebo-controlled study. Scand J Rheumatol 2004;33:94-101.

17 Kongsgaard M, Kovanen V, Aagaard P, et al. Corticosteroid injections, eccentric decline squat training and heavy slow resistance training in patellar tendinopathy. Scand J Med Sci Sports 2009;19:790-802.

18 Kearney RS, Parsons N, Metcalfe D, et al. Injection therapies for Achilles tendinopathy. Cochrane Database Syst Rev 2015;13.

19 van der Vlist AC, Winters M, Weir A, et al. Which treatment is most effective for patients with Achilles tendinopathy? A living systematic review with network meta-analysis of 29 randomised controlled trials. Br J Sports Med 2021:55:249-56.

20 Chan O, O'Dowd D, Padhiar N, et al. High volume image guided injections in chronic Achilles tendinopathy. Disabil Rehabil 2008;30:1697-708

21 Alfredson $\mathrm{H}$. Chronic midportion Achilles tendinopathy: an update on research and treatment. Clin Sports Med 2003;22:727-41.

22 Resteghini P, Yeoh J. High-volume injection in the management of recalcitrant mid-body Achilles tendinopathy: a prospective case series assessing the influence of neovascularity and outcome. Int Musculoskelet Med 2012;34:92-100.

23 Chaudhry FA. Effectiveness of dry needling and high-volume imageguided injection in the management of chronic mid-portion Achilles tendinopathy in adult population: a literature review. Eur J Orthop Surg Traumatol 2017;27:441-8.

24 Humphrey J, Chan O, Crisp T, et al. The short-term effects of high volume image guided injections in resistant non-insertional Achilles tendinopathy. J Sci Med Sport 2010;13:295-8.

25 Boesen AP, Hansen R, Boesen Ml, et al. Effect of high-volume injection, platelet-rich plasma, and sham treatment in chronic midportion Achilles tendinopathy: a randomized double-blinded prospective study. Am J Sports Med 2017;45:2034-43.

26 Boesen AP, Langberg $\mathrm{H}$, Hansen $\mathrm{R}$, et al. High volume injection with and without corticosteroid in chronic midportion Achilles tendinopathy. Scand J Med Sci Sports 2019;29:1223-31.

27 van der Vlist AC, van Oosterom RF, van Veldhoven PLJ, et al. Effectiveness of a high volume injection as treatment for chronic Achilles tendinopathy: randomised controlled trial. BMJ 2020;370:m3027.

28 Chan A-W, Tetzlaff JM, Altman DG, et al. SPIRIT 2013 statement: defining standard protocol items for clinical trials. Ann Intern Med 2013;158:200-7.

29 Munteanu SE, Scott LA, Bonanno DR, et al. Effectiveness of customised foot orthoses for Achilles tendinopathy: a randomised controlled trial. Br J Sports Med 2015;49:989-94.

30 Freynhagen R, Baron R, Gockel U, et al. Pain DETECT: a new screening questionnaire to identify neuropathic components in patients with back pain. Curr Med Res Opin 2006;22:1911-20.

31 Devilly GJ, Borkovec TD. Psychometric properties of the credibility/expectancy questionnaire. J Behav Ther Exp Psychiatry 2000;31:73-86.

32 Kolahi J, Bang H, Park J. Towards a proposal for assessment of blinding success in clinical trials: up-to-date review. Community Dent Oral Epidemiol 2009;37:477-84.

33 Malliaras P, Barton CJ, Reeves ND, et al. Achilles and patellar tendinopathy loading programmes. Sports Medicine 2013;43:267-86.

34 Malliaras P, Rodriguez Palomino J, Barton CJ, Palomino JR, Infographic BCJ. Infographic. Achilles and patellar tendinopathy rehabilitation: strive to implement loading principles not recipes. $\mathrm{Br} J$ Sports Med 2018;52:1232-3.

35 Silbernagel KG, Thomeé R, Eriksson Bl, et al. Continued sports activity, using a pain-monitoring model, during rehabilitation in patients with Achilles tendinopathy: a randomized controlled study. Am J Sports Med 2007;35:897-906.

36 Robinson JM, Cook JL, Purdam C, et al. The VISA-A questionnaire: a valid and reliable index of the clinical severity of Achilles tendinopathy. Br J Sports Med 2001;35:335-41.

37 Malliaras P, Chan O, Simran G, et al. Doppler ultrasound signal in Achilles tendinopathy reduces immediately after activity. Int $J$ Sports Med 2012;33:480-4

38 van der Vlist AC, Veen JM, van Oosterom RF, et al. Ultrasound Doppler flow in patients with chronic midportion Achilles tendinopathy: is surface area quantification a reliable method? $\mathrm{J}$ Ultrasound Med 2020;39:731-9. 
39 Kamper SJ, Maher CG, Mackay G. Global rating of change scales: a review of strengths and weaknesses and considerations for design. $J$ Man Manip Ther 2009;17:163-70.

40 Rabin R, de Charro F, Fd C. EQ-5D: a measure of health status from the EuroQol Group. Ann Med 2001;33:337-43.

41 Sallis JF, Haskell WL, Wood PD, et al. Physical activity assessment methodology in the Five-City Project. Am J Epidemiol 1985;121:91-106.

42 Lundberg MKE, Styf J, Carlsson SG. A psychometric evaluation of the Tampa scale for Kinesiophobia - from a physiotherapeutic perspective. Physiother Theory Pract 2004;20:121-33.

43 Sullivan MJL, Bishop SR, Pivik J. The pain catastrophizing scale: development and validation. Psychol Assess 1995;7:524-32.

44 Nicholas MK, McGuire BE, Asghari A. A 2-item short form of the Pain Self-efficacy Questionnaire: development and psychometric evaluation of PSEQ-2. J Pain 2015;16:153-63.
45 Mentiplay BF, Perraton LG, Bower KJ, et al. Assessment of lower limb muscle strength and power using hand-held and fixed dynamometry: a reliability and validity study. PLOS One 2015;10:e0140822.

46 Roos EM, Engström M, Lagerquist A, et al. Clinical improvement after 6 weeks of eccentric exercise in patients with mid-portion Achilles tendinopathy -- a randomized trial with 1-year follow-up. Scand J Med Sci Sports 2004;14:286-95.

47 Bouwmans $\mathrm{C}$, Krol M, Severens $\mathrm{H}$, et al. The iMTA productivity cost questionnaire: a standardized instrument for measuring and valuing health-related productivity losses. Value Health 2015;18:753-8.

48 van Hout B, Janssen MF, Feng Y-S, et al. Interim scoring for the EQ5D-5L: mapping the EQ-5D-5L to EQ-5D-3L value sets. Value Health 2012;15:708-15.

49 Ram R, Meeuwisse W, Patel C, et al. The limited effectiveness of a home-based eccentric training for treatment of Achilles tendinopathy. Clin Invest Med 2013;36:E197-206. 